\title{
A produção sobre divulgação científica na formação inicial de professores de
}

\section{Química}

\author{
Production on scientific dissemination in the initial education of Chemistry teachers \\ Producción sobre difusión científica en la formación inicial de profesores de Química
}

Recebido: 09/03/2021 | Revisado: 17/03/2021 | Aceito: 18/03/2021 | Publicado: 26/03/2021

\author{
Diane Ferreira da Silva Dapieve \\ ORCID: https://orcid.org/0000-0003-2720-5180 \\ Universidade Estadual do Oeste do Paraná, Brasil \\ E-mail: dianeferreira.s@hotmail.com \\ Dulce Maria Strieder \\ ORCID: https://orcid.org/0000-0003-4495-6664 \\ Universidade Estadual do Oeste do Paraná, Brasil \\ E-mail: dulce.strieder@unioeste.br \\ Marcia Borin da Cunha \\ ORCID: https://orcid.org/0000-0002-3953-5198 \\ Universidade Estadual do Oeste do Paraná, Brasil \\ E-mail: marciaborin@unioeste.br
}

\begin{abstract}
Resumo
Este trabalho tem como objetivo investigar a produção acadêmica com a temática divulgação científica (DC) na formação de licenciandos em Química, analisando os objetivos destes ao usar a DC, vantagens, limitações e lacunas sobre a temática e as perspectivas assumidas. A pesquisa é descritiva exploratória e teve como banco de dados a Biblioteca Digital Brasileira de Teses e Dissertações (BDTD), no período de 2010 a 2020. A seleção dos trabalhos se deu em duas etapas, leitura do título e resumo, e para os selecionados, foi realizada leitura na íntegra e análise descritiva. Os trabalhos desenvolvem a ideia de que a DC é um recurso que pode contribuir em diferentes aspectos na formação do professor e favorecer uma visão mais adequada sobre o processo de produção do conhecimento científico. Consideramos que a DC apresenta potencialidades para o ensino de Química e ao ser inserido em sala de aula favorece uma visão mais adequada sobre processo de produção da ciência. Realçamos a necessidade de mais trabalhos que que investigam a DC no ensino de Química.
\end{abstract}

Palavras-chave: Difusão da ciência; Ensino de química; Formação de professor.

\begin{abstract}
This work aims to investigate academic production involving scientific dissemination (SD) in the initial education of undergraduate Chemistry students, analyzing their objectives when using SD, advantages, limitations and gaps on the theme and perspectives. It's a descriptive and exploratory research and the database was the Brazilian Digital Library of Theses and Dissertations (BDTD), in the period from 2010 to 2020. The selection of works took place in two stages, title and summary reading, and for those selected, full assessment and descriptive analysis were performed. The work develops the idea that SD is a resource that can contribute in different aspects to teacher education and favor a more adequate view of the scientific knowledge production process. We believe that the SD has potential for Chemistry teaching and when it is inserted in the classroom, it favors a more adequate view on the science production process. We emphasize the need for more works that investigates SD in the teaching of Chemistry.
\end{abstract}

Keywords: Science diffusion; Chemistry teaching; Teacher training.

\section{Resumen}

Este trabajo tiene el objetivo de investigar la producción académica con el tema divulgación científica (DC) en la formación de estudiantes de licenciatura en Química, analizando sus objetivos al utilizar la DC, ventajas, limitaciones y lagunas sobre el tema y sus perspectivas. La pesquisa es exploratoria descriptiva y tuvo como base de datos la Biblioteca Digital Brasileña de Tesis y Disertaciones (BDTD), en el período entre 2010 y 2020. La selección de los trabajos ocurrió en dos fases, lectura de título y resumen, y para los seleccionados, se realizó lectura completa y análisis descriptivo. Los trabajos desarrollan la idea de que la DC es un recurso que puede contribuir en diferentes aspectos en la formación del profesor, favoreciendo una visión más adecuada del proceso de producción del conocimiento científico. Creemos que la DC presenta potenciales para la enseñanza de Química y, cuando insertada en el aula, favorece una visión más adecuada del proceso de producción de la ciencia. Destacamos la necesidad de más trabajos que investiguen la CD en la enseñanza de Química.

Palabras clave: Difusión de la ciencia; Enseñanza de química; Formación de profesores. 


\section{Introdução}

A Divulgação Cientifica (DC) é objeto de pesquisa de pesquisadores, como: Zamboni (2001), Grigoletto (2005), Ribeiro e Kawamura (2005), Nascimento (2008), Cunha (2009; 2019), Bueno (2010), Caldas (2010), Ferreira (2012), Gomes (2012), Rocha (2012), Dapieve (2016), Lima (2016), Vieira (2019) e muitos outros que se propõem a investigar os elos desta com o ensino de ciências e a sociedade.

A DC pode ser compreendida como um meio pelo qual a sociedade pode se informar sobre a ciência, tornando acessível os processos e conhecimentos produzidos pelos cientistas. Segundo Zamboni (2001), a divulgação científica pode ser compreendida como uma atividade de difusão da ciência, que é destinada a um contexto diferente do que a originou, e para isso, utiliza de diferentes recursos e técnicas para tornar públicas as informações da ciência e tecnologia para a sociedade em geral. Os recursos e técnicas tornam o discurso da divulgação científica acessível inclusive para o público "leigo", termo este relacionado a ter pouco conhecimento sobre determinado assunto ou tema da ciência.

Cunha (2009) apresenta que o termo DC pode ser definido como formas que o conhecimento científico pode ser difundido para sociedade, tornando esse conhecimento acessível para o público em geral. A difusão científica, de forma mais ampla, se daria por diferentes vias e recursos, de forma a comunicar informações da ciência para o público geral e/ou para especialistas.

Neste trabalho destacamos a inclusão da DC na formação inicial de professores em Química, pois é neste momento que o estudante terá contato com diferentes recursos e materiais que o ajudarão em sua profissão, assim, segundo Dapieve (2016), o acesso da DC na formação de professores se dá principalmente por iniciativas do docente em apresentar esse recurso aos estudantes. Ainda, Dapieve (2016) e Vieira (2019) têm indicado que o acesso aos meios da divulgação científica disponibilizado aos licenciandos podem influenciar significativamente no seu uso e forma de uso posterior na prática docente.

Para tanto, este trabalho teve como objetivo investigar a produção acadêmica resultante de pesquisas que se propõem a estudar a DC na formação inicial de professores em cursos de Química licenciatura, analisando pontos, como: Quais são os principais autores que fundamentam as pesquisas sobre a DC no Brasil? Quais são os principais objetivos de estudos sobre a DC nos cursos de Química licenciatura? Quais são as vantagens, limitações e lacunas sobre a temática apontadas nos trabalhos? Nas pesquisas com a DC, quais são as perspectivas assumidas sobre DC? Por meio, dessas questões a priori é que nosso olhar buscou tecer esse estudo.

\section{Metodologia}

A presente pesquisa se caracteriza como qualitativa do tipo descritiva exploratória. Para Gil (2002) a pesquisa descritiva possui o objetivo principal de descrever o fenômeno estudado e/ou estabelecer relações, podendo se aproximar da pesquisa exploratória quando os objetivos de estudo tendem a explorar os pontos de vistas e contribuir para construção de hipóteses.

Para nos aproximar do objetivo de fazer um levantamento bibliográfico sobre a divulgação científica na formação inicial de professores em Química no Brasil, no período de 2010 a 2020, necessitamos determinar inicialmente os termos de busca. Para tal, fizemos leituras de trabalhos de autores que investigam a DC, como: Ribeiro e Kawamura (2005), Nascimento (2008), Cunha (2009) e Ferreira (2012). Com a leitura dos trabalhos determinamos os termos: divulgação da ciência ou difusão da ciência ou popularização da ciência, que contém formação inicial ou ensino de ciências e ou educação Química.

Com a utilização dos termos iniciamos nossa busca por pesquisas disponíveis na Biblioteca Digital Brasileira de Teses e Dissertações (BDTD) e obtivemos um total de 84 trabalhos sobre a divulgação científica na formação inicial no ensino de ciências. No entanto, com a intenção de olhar os trabalhos voltados para a formação em Química licenciatura, foi necessário fazer uma nova seleção via leitura do título dos trabalhos observando o direcionamento para a área de concentração do estudo. 
A leitura dos resumos auxiliou a compreender os sujeitos envolvidos no estudo reconhecendo assim a amostra do estudo. Os trabalhos que não apresentavam tais informações foram desconsiderados. Usando os delineamentos propostos, a nossa amostragem final na plataforma BDTD foram quatro (04) pesquisas, sendo três (03) dissertações e uma (01) tese, conforme o Quadro 1 a seguir:

Quadro 1: Trabalhos sobre a divulgação científica na formação inicial de professores em Química licenciatura.

\begin{tabular}{|l|l|c|c|c|}
\hline \multicolumn{1}{|c|}{ Autor } & \multicolumn{1}{|c|}{ Título } & Tipo & Ano & Local \\
\hline Gomes, V. B. & $\begin{array}{l}\text { Divulgação Científica na Formação } \\
\text { Inicial de Professores de Química }\end{array}$ & Dissertação & 2012 & Distrito Federal \\
\hline Ferreira, L. N. A. & $\begin{array}{l}\text { Textos de Divulgação Científica para o } \\
\text { Ensino de Química: características e } \\
\text { possibilidades }\end{array}$ & Tese & 2012 & São Paulo \\
\hline Dapieve, D. F. S. & $\begin{array}{l}\text { A Divulgação Científica na Formação } \\
\text { Inicial de Professores em Química }\end{array}$ & Dissertação & 2016 & Paraná \\
\hline Vieira, A. C. & $\begin{array}{l}\text { Divulgação Científica: possibilidades de } \\
\text { inclusão na prática pedagógica de } \\
\text { professores de Química }\end{array}$ & Dissertação & 2019 & Paraná \\
\hline
\end{tabular}

Fonte: Autores.

Os trabalhos foram lidos na íntegra, buscando responder as indagações a que essa pesquisa se propôs investigar.

Os resultados aqui apresentados são advindos de uma pesquisa de tese, que está em construção e que possui aprovação do comitê de ética em pesquisa com seres humanos, registrada CAAE com o número 34323420.7.0000.0107.

\section{Resultados e Discussão}

Os dados aqui apresentados seguirão na mesma ordem do Quadro 1. O primeiro trabalho é uma pesquisa de Mestrado da autora Gomes (2012), intitulado como "Divulgação Científica na Formação Inicial de Professores de Química". O objetivo desta pesquisa foi investigar as potencialidades que os Textos de Divulgação Científica (TDC) apresentam para formação de professores em Química, para isso a autora elaborou sete (07) TDC, tendo como eixos norteadores: a experimentação, a natureza da ciência, a história e traços de ciência, tecnologia, sociedade e ambiente (CTSA). No que tange a escrita, a autora buscou usar uma linguagem que potencializasse uma interação dialógica-problematizadora em sala de aula. Para a produção dos textos, a autora se embasou no estudo de Ribeiro e Kawamura (2005), que destacam três elementos essenciais que caracterizam o discurso da divulgação científica: tema, estilo e composição.

Os TDC foram produzidos para alunos do curso de Química licenciatura da Universidade de Brasília (UnB) e da Universidade Estadual de Santa Cruz (UESC), e o estudo contou com 65 participantes (total). Os licenciandos realizaram leitura dos textos e responderam a um questionário que possibilitou que a autora pudesse analisar a relevância e potencialidades da DC na formação inicial. Como resultados obtidos, a autora considerou que os materiais da DC favorecem a formação de professores, contribuindo para repensar os recursos pedagógicos, estratégias didáticas e suas possibilidades. Ainda, para a autora, as atividades com TDC favorecem o desenvolvimento de práticas argumentativas e algumas vantagens como: contribuir para formação crítica, histórica e social do aluno e favorecer o conhecimento de recursos didáticos.

Em relação a lacunas sobre a temática investigada, a autora apontou algumas, como: poucas pesquisas que relacionam a formação de professores e o uso de TDC em contextos escolares; o atual modelo de formação inicial carente de reflexões sobre como e porque utilizar abordagens relativas a Natureza do Conhecimento $(\mathrm{NdC})$; ausência de estudos que investiguem 
atividades didáticas com TDC numa perspectiva investigativa, de modo a funcionar como elementos motivadores e estruturadores da aula; e, por fim, a necessidade de estudos sobre interações discursivas entre o futuro professor e o TDC. Sobre limitações do tema não foi possível perceber indicativos. Consideramos que a perspectiva adotada da DC nesse trabalho está vinculada a DC como recurso didático para a prática docente.

No que tange o conceito da DC, Gomes (2012) buscou compreendê-lo com base em alguns pressupostos teóricos explanados em produções como: Pasquali (1979); Massarani (1998); Silva (2004); Nascimento (2005); e Bueno (2008). Segundo a autora, Pasquali (1979) define a DC como uma recodificação da linguagem de forma a se tornar mais compreensível ao público em geral. Essa visão está em consonância com outros autores que fundamentaram o estudo, como Massarani (1998), que afirma que a DC é desenvolvida por cientista e/ou jornalistas e essa se caracteriza por uma descrição inteligível da ciência para a sociedade em geral. Segundo a autora, Bueno (2008) entende a DC como um processo de recodificação da linguagem científica especializada para uma linguagem acessível ao público geral. E, para tal, utiliza diferentes recursos, técnicas, veículos ou canais para divulgar o conhecimento científico.

Diante dos conceitos da literatura apresentados pela autora, Gomes (2012) se apoia na perspectiva apontada por Bueno (2008), para quem a DC é compreendida como uma recodificação da linguagem, na qual o conhecimento científico passa por uma transformação para o conhecimento escolar, caracterizando uma formulação do discurso para atender às atividades educacionais.

O segundo trabalho intitulado como "Textos de divulgação científica para o ensino de Química: características e possibilidades" é uma tese da autora Ferreira (2012). A pesquisa apresentou alguns objetivos, sendo eles: selecionar e analisar artigos da revista Ciência Hoje (CH) no período de 2004 a 2008, tendo como foco assuntos relacionados a Química; criar e analisar uma estratégia didática com textos de divulgação científica (TDC); realizar ações que possibilitassem analisar o conhecimento sobre as percepções e estratégias didáticas dos licenciandos em dinâmicas discursivas, usando TDC em sala de aula. Para compreender o funcionamento da leitura dos TDC pelos alunos, em condições de sala de aula, a autora utilizou a Análise do Discurso (AD) proposta por Orlandi (1996, 2000, 2002, 2009), principalmente sobre as noções de tipologia do discurso de autoria. Sobre o instrumento de análise dos TDC da revista $\mathrm{CH}$, a autora realizou uma adaptação no instrumento proposto pelas autoras Salém e Kawamura (1999) e Ribeiro e Kawamura (2005). O primeiro referencial trouxe elementos sobre a utilização da DC no ensino de Física, e o segundo forneceu um instrumento de análise, baseado em algumas categorias vinculadas ao conteúdo e forma. Com base nos trabalhos a autora realizou algumas adaptações, adicionando novas subcategorias a partir da análise dos textos.

Para compreender o processo de pesquisa proposto por Ferreira (2012), torna-se necessário olharmos para seu percurso metodológico, o qual apresentaremos de forma sucinta. A autora tomou como ponto inicial da coleta de dados a revista Ciência Hoje $(\mathrm{CH})$ publicados no período de 2004 a 2008, o que gerou aproximadamente 50 textos. Os artigos selecionados foram analisados segundo o conteúdo e forma, adaptado de Salém e Kawamura (1999) e Ribeiro e Kawamura (2005), conforme já explanado anteriormente, e depois os mesmos textos foram analisados de acordo com as características discursivas, como cientificidade, laicidade e didaticidade. A coleta dos dados com licenciandos da formação inicial, se deu na disciplina de Prática do Ensino de Química, ofertada para alunos do último ano do curso de Licenciatura em Química. Nessa disciplina os alunos realizam o estágio supervisionado, o que permitiu Ferreira (2012) observar os licenciandos usando o TDC em situação de ensino. Como os alunos teriam que utilizar os TDC, Ferreira (2012) ministrou uma aula sobre TDC, de modo que, os acadêmicos tivessem contato e conhecimento de suas características e conseguissem elaborar o projeto de suas regências usando o TDC. Como última etapa da coleta de dados, os licenciandos fizeram um relatório do estágio e participaram de uma entrevista semiestruturada.

Deste modo, os resultados que Ferreira (2012) apresenta são um recorte da análise do discurso de alguns pontos, 
como: das interações entre alunos e professor durante a atividade de leitura e discussão do TDC; respostas dos alunos ao questionário sobre o TDC e dos textos produzidos pelos acadêmicos na regência; e o recorte analisado de pesquisas nacionais sobre o tema. Segundo Ferreira (2012), as pesquisas sobre TDC têm se voltado a investigar a inserção desses textos no ensino, de forma a ter uma melhor compreensão das suas finalidades e possiblidades. No ensino, os estudos tendem a investigar a sua inserção como recurso didático em diferentes perspectivas, por parte do professor e do aluno. A maioria das estratégias didáticas encontradas em seu estudo, se direcionam para a análise de textos. Alguns trabalhos sugerem aprofundamento de discussões sobre a inserção dos TDC em sala de aula e, também, da preparação do professor para o seu uso. Ferreira (2012) também aponta poucos trabalhos na área de conhecimento da Química.

Nas análises dos TDC da revista CH, Ferreira (2012) observou que estes apresentam aspectos da natureza da ciência, o que contribui para uma melhor compreensão do processo de construção da ciência.

Sobre os textos escolhidos pelos alunos para a regência, a autora observou que a maioria não atendia as características de um TDC. Durante a regência, os textos assumiram algumas funções, como: instigar o interesse dos alunos; evidenciar o desenvolvimento científico e tecnológico; incentivar a contextualização dos conteúdos; incentivar o hábito da leitura; possibilitar que os alunos tenham conhecimento da linguagem científica; contextualizar o conhecimento químico; favorecer o entendimento e explicação dos conceitos; e favorecer uma interação entre professor e aluno. Assim, Ferreira (2012) acredita que os licenciandos apresentaram apropriação dos temas relativos aos TDC em sala de aula, e sinaliza contribuições do processo da formação acadêmica na prática dos alunos. Sobre a autoria, segundo a autora, adotando o viés de Orlandi (1996, 2000, 2002, 2009), foi observado durante a regência a mudança do discurso autoritário para o discurso polêmico.

As vantagens apontadas pela autora sobre os TDC estão no sentido das contribuições para a formação do aluno em diferentes aspectos e para melhor compreensão sobre a ciência.

A autora deixa claro lacunas que precisam ser revistas, como: os sentidos produzidos em situações de ensino em que esteja presente o uso TDC em diferentes graus de didaticidade, cientificidade e laicidade. Outro indicativo é a necessidade de pesquisas que busquem investigar como os estudantes se apropriam dos conceitos Químicos a partir do uso dos TDC.

Sobre possíveis limitações do uso de TDC, a autora não faz afirmações de forma explícita, mas da nossa análise sobre sua pesquisa, é possível indicar limitações das estratégias utilizadas pelos alunos, sendo que a maioria usou os TDC para desenvolver a experimentação em sala de aula, não sendo, portanto, o foco principal direcionado ao texto. O segundo indicativo está relacionado a carência de produções encontradas no levantamento de pesquisas nacionais envolvendo a DC na área de conhecimento do ensino da Química. Pela sua busca essa área é que apresentou menor frequência de trabalhos, fomentando a necessidade de pesquisas como a que ela se propôs em sua tese.

No que tange aos indicativos de perspectivas adotadas sobre a DC, além de recurso didático foi possível notar uma tendência para formação crítica.

A construção do conceito pela autora Ferreira (2012), se deu pelas contribuições de alguns autores como: Zamboni (2001); Mora (2003); Baalbaki (2006); Cunha; Giordan (2009). A autora considera a DC como difusão do conhecimento científico para público geral, que consiste na formulação de um novo discurso com características próprias da sua produção.

O terceiro trabalho, intitulado “A Divulgação Científica na Formação Inicial de Professores de Química”, é de autoria de Dapieve (2016), que teve como objetivo investigar a compreensão que estudantes do curso de Licenciatura em Química possuem acerca da divulgação científica e sobre a sua contribuição como recurso didático no ensino de Química. De modo a atingir os objetivos pretendidos, o primeiro passo foi determinar a amostra de participantes da pesquisa, composta por acadêmicos do último ano do curso de Química Licenciatura de uma universidade pública do sul do Brasil. A autora partiu do pressuposto de que esses alunos, por estarem finalizando o curso, possuiriam conhecimentos sobre metodologias e recursos didáticos que foram vivenciados no seu processo de formação inicial e, além disso, já teriam concluído ou estariam concluindo 
o estágio supervisionado. A realização do estágio supervisionado permitiria a autora evidenciar relações e influências das experiências advindas da formação inicial com a sua prática no estágio supervisionado e, assim, identificar as potencialidades e contribuições da DC.

Determinado os sujeitos da amostra, o segundo passo foi investigar a compreensão dos licenciandos sobre o tema de pesquisa. Isto se deu via questionário entregue e respondido por treze (13) acadêmicos. O questionário, também serviu como instrumento de seleção para a última etapa da pesquisa, a entrevista. Foram selecionados para a entrevista quatro (04) licenciandos, que afirmaram ter usado ferramentas da DC em seus estágios supervisionados.

Os resultados demonstram que os licenciandos possuem uma certa compreensão sobre o que é a DC e para quem ela se destina, considerando a DC como algo importante para ampliar o conhecimento sobre os avanços científicos e tecnológicos. Em relação a forma como foi trabalhada a DC na formação inicial dos participantes, a mais expressa foi a leitura de textos, o que refletiu, segundo a autora, nas propostas dos alunos nos estágios supervisionados, onde a maioria fez uso desta mesma metodologia, evidenciando como as experiências advindas da formação inicial influenciam na forma com que os estudantes vão trabalhar com o recurso. No que tange aos meios de DC relevantes para o ensino de Química, foram citados: a internet e revistas, sendo esses os meios didáticos mais utilizados pelos licenciandos.

Sobre as vantagens da DC advindas da pesquisa, estão: proporcionar a atualização do conhecimento, favorecer o interesse do aluno, evidenciar a provisoriedade da ciência e contribuir para a formação crítica.

Em relação as lacunas e limitações, a autora não deixou de forma explícita esses tópicos apontados no interior do texto, mas foi possível apontar, com base na leitura, alguns indícios de limitações associados a forma de trabalho com DC e seu uso em sala de aula, no qual a maioria das propostas apresentou uma certa tendência a práticas simplistas e pragmáticas da ciência.

Sobre a perspectivas da DC presentes, é possível perceber uma tendência da DC crítica e reflexiva no processo de ensino e aprendizagem.

O conceito construído pela autora, que compreende a divulgação científica como nova formulação discursiva e que se destina a atender ao público geral, foi alicerçado por referenciais como: Zamboni (2001); Nascimento (2008); Cunha (2009); Ferreira (2012).

Por fim, o quarto e último trabalho aqui analisado é a dissertação de Vieira (2019), intitulada "Divulgação Científica: possibilidades de inclusão na prática pedagógica de professores de química". A pesquisa teve como objetivo investigar se estudantes do curso de Química Licenciatura de uma universidade pública utilizaram a DC em projeto de ação didática (PAD) e, se o fizeram, quais eram os propósitos de ensino e, ainda, se após a formação inicial, estes continuaram a utilizar a DC em suas aulas.

Para a seleção da amostra a autora escolheu o período de 2014 a 2016, pois a inclusão do PAD aconteceu no Projeto Político Pedagógico do curso a partir de 2014. O acesso aos PADs foi por meio do CD entregue pela coordenação do curso. Além da análise do PAD, a autora também analisou o relatório final desse projeto. A intenção de analisar os dois (PAD e relatório) foi de identificar se os alunos inseriam a DC de forma proposital ou se a DC estava presente de forma indireta. Para isso, Vieira (2019) se baseou no estudo de Lima e Giordan (2015) e Lima (2016), que apresenta alguns propósitos da DC para o ensino, sendo eles: contextualização histórica, explicação, levantamento de concepções, metacognição, pesquisa, produção de material, promoção do debate e trabalho de campo. Munida do referencial teórico, a autora analisou vinte e sete (27) PADs, que apresentavam alguns propósitos da DC. Com essa etapa, a autora também realizou entrevistas com seis (06) egressos do curso, que possuíam experiência de docência em sala de aula, de modo a atingir os objetivos propostos no trabalho. Para a análise dos dados foram utilizados pressupostos teóricos da análise de discurso (AD) de Orlandi.

Os resultados apresentados pela autora demonstram que, na maioria dos PADs havia propósitos claros e definidos 
para usar a DC, em outros os propósitos não estavam claros, mas estavam presentes. Outros resultados presentes no trabalho foram: o contato da DC na disciplina da formação inicial contribuiu para conhecimento e compreensão sobre a DC; a DC permite trabalhar assuntos atuais facilitando as atividades escolares; os entrevistados consideraram os TDC como um recurso importante para o ensino; e os PADs demonstraram pouca exploração das atividades envolvendo a DC, assim como em atividades com TDC. De modo geral, a autora identificou que eram feitas análises superficiais do TDC, em especial a utilização de grifos de destaque no texto por parte do estudante. A pesquisa de Vieira (2019) nos mostra que a forma como o professor trabalha com a divulgação científica ainda está muito vinculada ao ensino tradicional, deste modo o professor renova os recursos, mas altera pouco em sua prática em sala de aula.

É possível perceber pela pesquisa de Vieira (2019) que a inserção da DC na formação inicial influenciou o seu uso na prática docente dos entrevistados, sendo notável uma similaridade nas formas propostas de trabalho em sala de aula com o que foi vivenciada no processo de formação.

Em relação as vantagens do uso dos materiais de DC em sala de aula, a autora aponta o desenvolvimento de habilidades e competências e favorecimento da alfabetização científica. Também aponta que este enriquece as aulas, proporciona momentos de discussão e contribui para a atualização do professor sobre temas da ciência.

No que se refere ao item lacunas do tema, a autora chama a atenção para a visão que é apresentada sobre a DC enquanto facilitadora e pragmática.

A autora apresenta que as limitações em se trabalhar com a DC em sala de aula observadas em seu trabalho foram essencialmente o fator tempo e a forma de trabalhar com a DC. Em relação ao fator tempo indica está ligado com o preparo dos materiais de DC para sala de aula, sendo que muitos não dispões de tempo para planejar da forma que gostariam e também pelo grande número de turmas. Já a forma de trabalhar com a DC está muito vinculada ao aspecto pragmático de trabalho.

Sobre a perspectiva que se manifesta nesse trabalho, nota-se indicativos vinculados a formação de habilidades e competências do aluno para trabalhar com recursos da DC em sala de aula. Esses indícios são evidenciados durante a leitura do trabalho, mas também nas possibilidades de trabalhar a DC na formação apontadas pela pesquisadora, como: a permanente e efetiva leitura de TDC nas aulas da graduação; a realização de atividades práticas com o uso da DC em disciplinas; a inclusão de atividades externas à universidade; atividades didáticas durante a formação; a elaboração de textos de divulgação da ciência por parte dos acadêmicos; leituras e discussões de livros que divulgam a ciência; a leitura de textos originais, favorecendo a construção uma imagem mais adequada da ciência; espaço de leitura; a proposição de atividades de pesquisa usando a internet.

A autora não apresenta um conceito de DC construído na sua pesquisa, mas menciona enquanto alicerces de sua visão de DC alguns autores, como: Bueno (1985); Zamboni (1997); Massarani (1998); Silva (2006); Nascimento (2008); Cunha (2009); Grillo (2013); Nascimento (2015). Estes autores trazem diferentes compreensões da DC, mas estão alinhados sobre a DC se destinar ao público geral.

\section{Conclusão}

O presente trabalho buscou investigar a produção acadêmica cujo tema se remete a estudar a DC na formação inicial de professores em cursos de Química licenciatura. As análises foram norteadas pelas questões: Quais são os principais autores que fundamentam as pesquisas sobre a DC no Brasil? Quais são os principais objetivos de estudos sobre a DC nos cursos de Química licenciatura? Quais são as vantagens, limitações e lacunas sobre a temática apontadas nos trabalhos? Nas pesquisas com a DC, quais são as perspectivas assumidas sobre DC?

Com base nos resultados apresentados, vemos que há muitos autores que fundamentam e colaboram com as pesquisas sobre a DC no ensino de Química, e alguns autores se sobressaíram mais em relação a outros, como: Nascimento (2005, 2008, 2015); Bueno (2008), Zamboni (2001); Cunha (2009) e Ferreira (2012). 
Em relação a segunda questão da pesquisa sobre os objetivos das pesquisas que se propõem a estudar a DC, esses versam sobre as contribuições e potencialidades para formação do estudante. Já sobre as vantagens, limitações e lacunas, alguns autores indicaram de forma clara ao longo do trabalho e outros não apresentam de forma explícita, mas foi possível fazer inferências com base na leitura. Dentre esses ressaltamos a lacuna apresentada pela autora Ferreira (2012), sobre a necessidade de pesquisas que investiguem como os estudantes se apropriam dos conceitos Químicos com o uso dos TDC.

Em relação aos conceitos sobre a DC, houve divergência entre as produções analisadas. Para Gomes (2012), a DC é compreendida uma recodificação da linguagem, no qual o conhecimento científico passa por uma transformação para o conhecimento escolar, em um processo semelhante à transposição didática, enquanto, para Ferreira (2012), Dapieve (2016) e Vieira (2019), a DC é a formulação de um novo discurso com características próprias da sua produção, isto é, um novo gênero de discurso.

Com a realização deste trabalho, percebemos o quanto as atividades desenvolvidas dentro do processo de formação inicial influenciam nas práticas dos licenciandos, o que nos faz refletir ainda mais sobre como é necessário olhar para os professores formadores, pois esses servirão como referência para as ações docentes de seus alunos.

A partir deste contexto, apontamos a necessidade de ampliação das pesquisas que investigam o processo da inclusão da DC na formação inicial de professores em Química licenciatura, com destaque para análises que abarquem também as percepções e formação dos formadores atuantes nestes cursos, frente aos diversos meios da DC.

\section{Referências}

Bueno, W. C. (2010). Comunicação científica e divulgação científica: aproximações e rupturas conceituais. Informação \& Informação, Brasília, 15, 1-12.

Caldas, G. (2010). Mídia, Educação Científica e Cidadania: a experiência das revistas Eureca e ABC das Águas. In: Pinto, G. A. (Org.). Divulgação científica e práticas educativas. Curitiba: CRV, 149 - 166.

Cunha, M. B. (2009). A percepção de Ciência e Tecnologia dos estudantes de Ensino Médio e a divulgação científica. 364f. Tese (Doutorado em Educação) Universidade de São Paulo, São Paulo.

Cunha, M. B. (2019). Divulgação científica: diálogos com o ensino de Ciências. Appris, 189.

Dapieve, D. F. S. (2016). A divulgação científica na formação inicial de professores em Química. 125 f. Dissertação (Mestrado em Educação para Ciências e Matemática) - Universidade Estadual de Maringá, Maringá.

Ferreira, L. N. A. (2012). Textos de Divulgação Científica para o Ensino de Química: Características e Possibilidades. 304f. Tese (Doutorado em Ciências) Universidade Federal de São Carlos, São Carlos.

Gil, A. C. (2002). Como elaborar projetos de pesquisa. (4a ed.), Atlas.

Gomes, V. B. (2012). Divulgação Científica na Formação Inicial de Professores de Química. 178f. Dissertação (Mestrado Profissional em Ensino de Ciências) - Universidade Brasília, Brasília.

Grigoletto, E. (2005). O Discurso de Divulgação Científica: um espaço discursivo intervelar. 269f. Tese (Doutorado em Letras) - Universidade Federal do Rio Grande do Sul, Porto Alegre.

Lima, G. (2016). O professor e a divulgação científica: apropriação e uso em situações formais de ensino. 311f. Tese de Doutorado. Programa de PósGraduação em Educação, Ensino de Ciências e Matemática. Faculdade de Educação da Universidade de São Paulo.

Nascimento, T. G. (2008). Leituras de Divulgação Científica na Formação Inicial de Professores de Ciências. 376f. Tese (Doutorado em Educação Científica e Tecnológica) - Universidade Federal de Santa Catarina, Florianópolis.

Ribeiro, R. A. \& Kawamura, M. R. (2005). A ciência e diferentes vozes: uma análise de textos de divulgação científica. In Encontro Nacional de Pesquisa em Educação em Ciências, 5., Bauru. Atas... Bauru.

Rocha, M. B. (2012). O potencial didático dos textos de divulgação científica segundo professores de ciências. R.B. E. C. T., 5 (2), $47-68$.

Vieira, A. C. (2019). Divulgação Científica: possibilidades de inclusão na prática pedagógica de professores de Química. Dissertação (Mestrado em Educação) - Universidade Estadual de Oeste do Paraná, Cascavel.

Zamboni, L. M. S. (2001). Cientistas, jornalistas e a divulgação científica: subjetividade e heterogeneidade no discurso da divulgação científica. Autores Associados. 